\title{
Enhanced backscattering by multiple nanocylinders illuminated by TE plane wave
}

\author{
Hao-Yuan She ${ }_{3}^{1}$ Le-Wei Li, ${ }^{1,3, a)}$ Soo Jin Chua, ${ }^{1}$ Wei-Bin Ewe, ${ }^{2}$ Olivier J. F. Martin, ${ }^{3}$ and \\ Juan R. Mosig \\ ${ }^{1}$ Nanoscience and Nanotechnology Initiative and Department of Electrical and Computer Engineering, \\ National University of Singapore, Kent Ridge 119260, Singapore \\ ${ }^{2}$ Institute of High Performance Computing, Singapore Science Park II 117528, Singapore \\ ${ }^{3}$ Institute for Transmission, Waves and Photonics, Swiss Federal Institute of Technology, Lausanne \\ CH-1015, Switzerland
}

(Received 4 June 2008; accepted 28 June 2008; published online 24 September 2008)

\begin{abstract}
In this paper, we study the multiple scattering by electrically small (the radius of the cylinder is much smaller than the wavelength) plasmonic nanocylinders near surface plasmon resonance. The cylinders are assumed to be identical in dimension and composition. The incident plane wave is assumed to be TE polarized so that the plasmon resonance of two-dimensional cylindrical structures (for both individual and group of cylinders) can be excited. It is found that multiple plasmonic cylinders enhance the near-field magnetic field intensity due to mutual coupling. When the electrical dimension $q$ of the cylinders $\left(q=k_{0} R\right.$, where $k_{0}$ is the wave number of the free space and $R$ is the radius of the cylinder) is fixed, the magnitude of the field distribution primarily depends on the positions of the cylinders at normal incidence. (C) 2008 American Institute of Physics. [DOI: $10.1063 / 1.2975214]$
\end{abstract}

\section{INTRODUCTION}

Light scattering by two-dimensional photonic crystal structures has been a classical subject of scientific and academic interests for many years. The scattering properties of an array of parallel cylinders randomly distributed were studied in Ref. 1. Periodic structures also attracted a lot of interests, ${ }^{2-4}$ and Yasumoto and Yoshitomi $^{5}$ developed Green's function for periodic two-dimensional photonic crystals. Analysis of photonic crystals has also been discussed by an effective-index method. ${ }^{6}$ With the development of nanoscience and nanotechnology and with some progresses in nanoscience, property studies of light scattering by nanoscaled particles or objects remain as an important topic of increasing interest in the recent few years. For a single two-dimensional electrically small nanocylinder, it is well known that the surface plasmon resonance happens when the real part of the relative permittivity of the cylinder, $\mathfrak{R e}\left(\epsilon_{r}\right)=-1$, and Luk'yanchuk and Ternovsky discussed the problem thoroughly in Ref. 7. Evidently, the near-field energy intensity and the total cross section can be enhanced significantly. This phenomenon happens when the incident wave is a TE-polarized plane wave. In this case, the Rayleigh scattering for small arguments of the lossless scatterers cannot be used usually because it will theoretically allow a nanocylinder to have an infinitely large cross section, which is known as the divergence problem. For the low damping cases, the exact solutions, i.e., the Mie scattering theory, should be applied to calculate the optical properties. Enhanced backscattering of a metallic nanocylinder with a random rough surface was studied using the method of stochastic functional approach. ${ }^{8}$ The incident plane wave is assumed

${ }^{a)}$ Electronic mail: 1wli@nus.edu.sg. URL: http://www.ece.nus.edu.sg/lwli. to be a TE-polarized plane wave and the surface plasmon waves were found to contribute considerably to the enhanced backscattering. Backscattering enhancement of light by nanoparticles has also been reported. ${ }^{9}$ For multiple scatterers, optical properties of metallic nanorod arrays embedded in alumina were presented and the optical transmittance was measured as a function of relative parameters. ${ }^{10}$ Gumbs and Aizin $^{11}$ discussed the collective plasma excitations of a linear periodic array of two-dimensional multilayered cylindrical nanotubes. The operation of laser in the visible regime at room temperature in two-dimensional photonic crystal membrane was studied in Ref. 12. The surface plasmon polaritons of metallic coated cylinders with dielectric core were presented in Ref. 13. The detection of the optical magnetic field for dielectric samples in the near-field region was discussed in Ref. 14. The experimental and theoretical studies of optical properties of metallodielectric periodic structures with subwavelength slots were given in Ref. 15. The localized defect modes in two-dimensional photonic crystals with an isolated defect were analyzed by means of the multiple scattering theory in Ref. 16. The spectra of plasma and electromagnetic waves of two-dimensional parallel thin metallic cylinders in dielectric media are calculated. ${ }^{17} \mathrm{~S}$-wave resonance mode with $m=0$ by $\mathrm{Si}$ nanowires was shown in Ref. 18. The scattering matrix method (SMM) is utilized to deal with the problem of three-dimensional anisotropic photonic crystal slabs. ${ }^{19}$ The optical properties of nanocylinder arrays were studied analytically in Ref. 20. The second-harmonic emission in two-dimensional photonic crystals was investigated numerically using the multiple scattering method in Ref. 21. Metallic waveguide structures were also studied in the role of the plasmonic modes theoretically, ${ }^{22}$ and nanosilicon waveguide is analyzed in Ref. 23.

Properties of light transmission through photonic crystal 


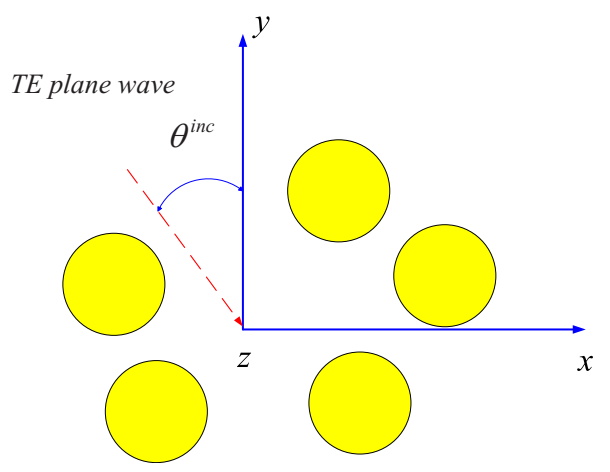

FIG. 1. (Color online) Geometry for the scattering of a plane wave by multiple cylinders in free space.

structures were also discussed for arrays of subwavelength periodic holes, ${ }^{24}$ enhanced transmission due to cylindrical surface plasmons, ${ }^{25}$ extraordinary optical reflection by subwavelength cylinder arrays, ${ }^{26}$ transmission properties of light in two-dimensional structures, ${ }^{27-29}$ and guided resonances in three-dimensional photonic crystal slab structures. ${ }^{30}$ The extraordinary optical transmission without plasmons in the $s$-polarization case was also addressed in Ref. 31 . The negative index metamaterials were studied in Refs. 32-43 experimentally and theoretically. The evidence for positive refraction of two-dimensional photonic crystal structures was demonstrated in Ref. 44. The negative refraction of chiral media was discussed in Refs. 45 and 46. We have also discussed the surface polaritons of coated cylindrical structures ${ }^{47}$ and focusing surface plasmons by changing the incident angle are shown in Ref. 48.

These are some interesting optical properties exhibited by two-dimensional photonic crystal structures. In this paper, we will therefore characterize the enhanced backscattering by multiple nanocylinders under plasmon resonance. The multiple scattering problems are solved using the SMM. We have analyzed finite arrays with different distributions. The incident wave is assumed to be in the normal direction. Several cases have been taken into consideration and distributions of the near-field magnetic field intensity are clearly shown.

\section{THEORETICAL FOUNDATION}

The geometry of the problem is shown in Fig. 1. The incident wave is assumed to be a TE plane wave whose magnetic field is polarized in the $z$-direction (i.e., along the axis of the cylinder) and it can be expressed as

$$
H_{z}^{\mathrm{inc}}=H_{1}^{i}=H_{0} \sum_{m=-\infty}^{\infty} i^{m} J_{m}\left(k_{0} \rho\right) e^{i m \theta^{\mathrm{inc}}}
$$

The total scattered field is obtained by summing the fields scattered by the entire set of cylinders and is given by

$$
H_{z}^{\mathrm{sc}}=H_{0} \sum_{j=1}^{N} \sum_{m=-\infty}^{\infty} b_{j, m} H_{m}^{(1)}\left(k_{0} r_{j}\right) e^{i m \theta_{j}},
$$

where $H_{0}$ denotes the amplitude of the incident magnetic field, $k_{0}$ stands for the wave number of the free space, $r_{j}$ represents the distance from the center of the $j$ th cylinder to the observing point, and $N$ is the total number of the cylinders. The scattering coefficient $b_{j, m}$ can be expressed by

$$
b_{j, m}=A_{m} a_{j, m},
$$

where the coefficient $A_{m}$ is defined as

$$
A_{m}=\frac{\sqrt{\epsilon_{r}} J_{m}\left(\sqrt{\epsilon_{r}} q\right) J_{m}^{\prime}(q)-J_{m}^{\prime}\left(\sqrt{\epsilon_{r}} q\right) J_{m}(q)}{\sqrt{\epsilon_{r}} J_{m}\left(\sqrt{\epsilon_{r}} q\right) H_{m}^{(1)^{\prime}}(q)-J_{m}^{\prime}(q) H_{m}^{(1)}\left(\sqrt{\epsilon_{r}} q\right)},
$$

while $q_{j}=k_{0} R_{j}$, where $R_{j}$ denotes the radius of the $j$ th cylinder, $J_{m}(\bigcirc)$ stands for the cylindrical Bessel function of the first kind and the order $m$, and $H_{m}^{(1)}(\bigcirc)$ represents the cylindrical Hankel functions of the first kind and order $m$. Here we assume that the cylinders are identical in dimension, so $R_{j}=R$. The other coefficient $a_{j, m}$, which introduces the coupling effects of the cylinders, is defined as

$$
\begin{aligned}
a_{j, m}= & (-i)^{m} e^{i k_{0} r^{j} \sin \left(\theta^{\mathrm{inc}}-\theta^{j}\right)-i m \theta^{\mathrm{inc}}}+\sum_{l \neq j} \sum_{p=-\infty}^{\infty} e^{i(p-m) \theta_{l}^{j}} H_{m-p}^{(1)} \\
& \times\left(k_{0} r_{l}^{j}\right),
\end{aligned}
$$

where $r^{j}$ and $r_{l}^{j}$ denote the distances between the center of the $j$ th cylinder and the original point and the centers between the $j$ th and $l$ th cylinders, respectively. The other symbols as shown above have the same meanings as those given in Ref. 1 and will not be given herein. With these linear equations obtained, we can solve them for the scattering coefficients.

\section{SCATTERING PROPERTIES OF A SINGLE PLASMONIC CYLINDER}

For a single cylinder in free space, $A_{m}$ represents the scattering coefficient and it depends on the composition and geometry of the cylinder. The values of the coefficient can affect significantly both the near- and far-field characteristics. As given in Ref. 7, we have the eigenvalue symmetry of the coefficients $A_{m}=A_{-m}$. Therefore, we only consider the case for $m>0$.

Plotted in Fig. 2 is the real part of $A_{1}$ versus $k_{0} R=0.1$, where $k_{0} R=0.2$ and different damping terms are considered.

The relative permittivity is assumed to be $\epsilon_{r}=\epsilon^{\prime}+i \epsilon^{\prime \prime}$. It is clearly seen that as electrical dimensions increase, the damping effect will be less important for the scattering coefficients at the resonance. For nonabsorbing cylinders, the amplitude of $\left|\mathfrak{R e}\left(A_{m}\right)\right|$ can always reach 1 . This is where the plasmon resonance usually happens for cylindrical structures. It is apparent that the position of the resonances will not change when different damping terms are considered. For the higher order modes, even a very small damping term will affect the scattering coefficients significantly as shown in Fig. 3.

We can hardly observe any plasmon resonance properties in Fig. 3 for those with $\epsilon^{\prime \prime}=0.01$ and $\epsilon^{\prime \prime}=0.1$. In Fig. 4, the resonance properties of $\mathfrak{R e}\left(A_{m}\right)$ are shown versus the real part of $\epsilon_{r}$ with different electrical dimensions varying from 0.1 to 0.3 . The lossless and the dissipative cases are considered in Fig. 4 for comparison purposes. 


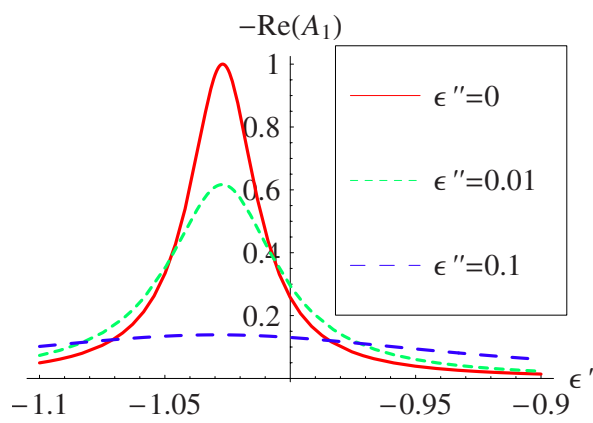

(a)

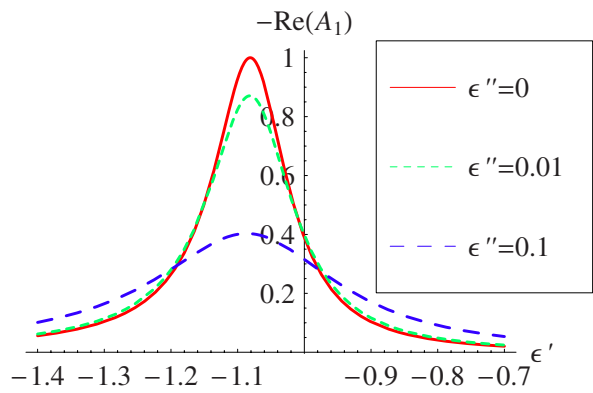

(b)

FIG. 2. (Color online) $\mathfrak{R e}\left(A_{1}\right)$ vs $\mathfrak{R e}\left(\boldsymbol{\epsilon}_{r}\right)$ for different electrical dimensions $q$ and damping terms $\operatorname{Im}\left(\epsilon_{r}\right)$

The total magnetic field intensity distribution outside of a lossless nanocylinder scattered by a TE plane wave with an amplitude of $H_{0}=1$ is expressed as $\left|H_{z}^{\text {total }}\right|$ and is shown in Fig. 5(a).

The wavelength is $366 \mathrm{~nm}$ near the visible region. The radius of the nanocylinder is $17.5 \mathrm{~nm}$. The relative permittivity of the nanocylinder is assumed to be -1.14 , which is near the plasmon resonance as shown in Fig. 4 where the incident angle is $\pi / 2 \mathrm{rad}$. It is clear that the backscattering intensity of the near field can be enhanced significantly. In Fig. 5(b), we consider a plasmonic nanocylinder with the
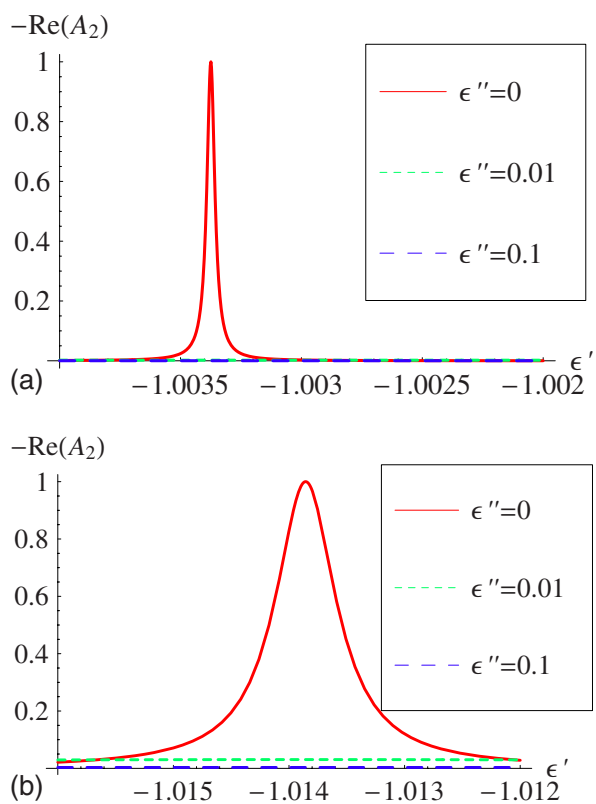

FIG. 3. (Color online) $\mathfrak{R e}\left(A_{2}\right)$ vs $\Re \mathrm{e}\left(\epsilon_{r}\right)$ for different electrical dimensions $q$ and damping terms $\operatorname{Im}\left(\epsilon_{r}\right)$.

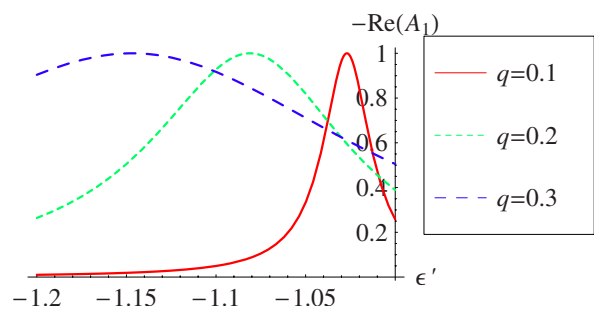

(a)

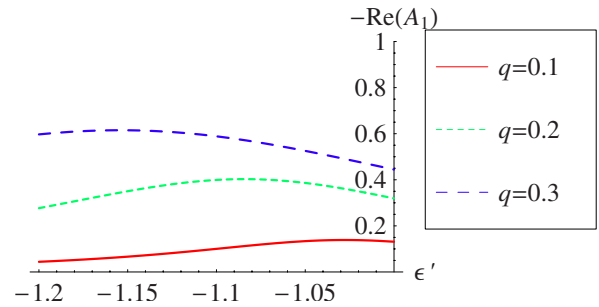

(b)

FIG. 4. (Color online) $\mathfrak{R e}\left(A_{1}\right)$ vs $\mathfrak{R e}\left(\epsilon_{r}\right)$ for damping term $\mathfrak{R m}\left(\epsilon_{r}\right)$ and different electrical dimensions $q$.

relative permittivity of $\epsilon_{r}=-1.14+0.1 i$. The other parameters are the same as those shown in Fig. 5(a) and will not be repeated herein. It is seen that although the total magnetic field intensity decreases as compared to the lossless case

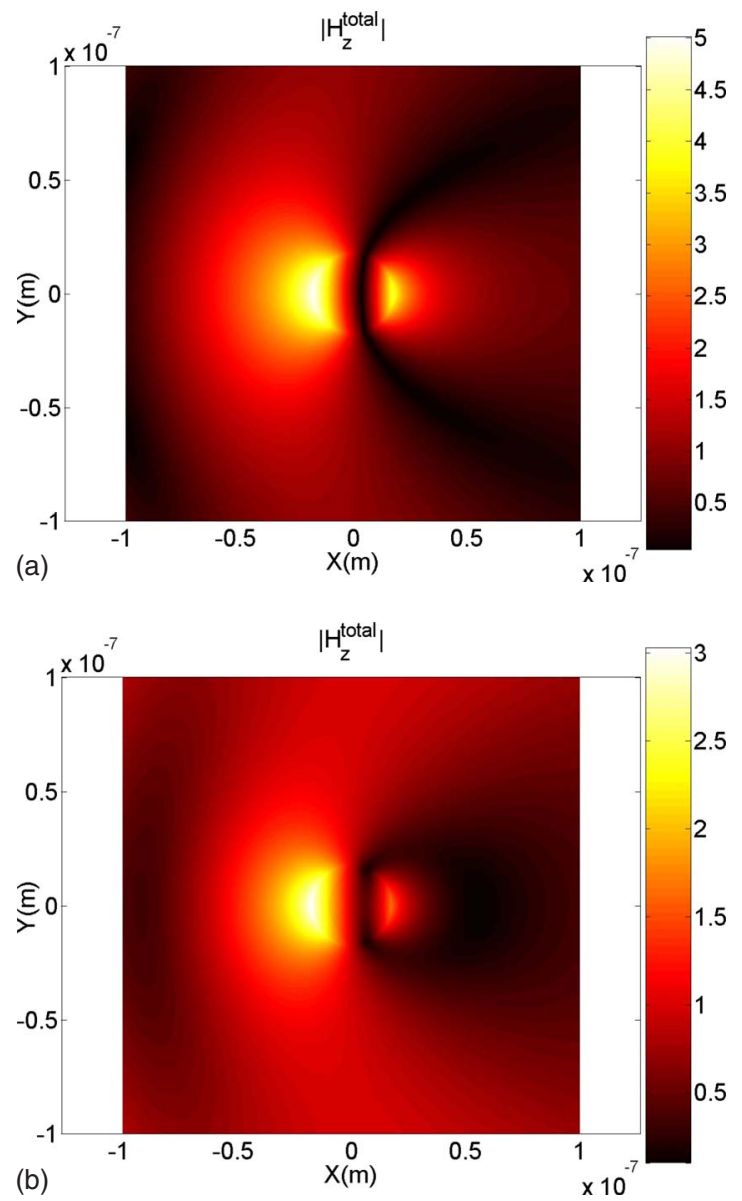

FIG. 5. (Color online) Scattering by a single plasmonic cylinder with different relative permittivities. The wavelength is $366 \mathrm{~nm}$ and the radius of the cylinder is $17.5 \mathrm{~nm}$. In (a) we present the lossless case and in (b), the damping term is considered. 
shown in Fig. 5(a), the backscattering is still very strong and the field intensity (defined as $I=\boldsymbol{H} \cdot \boldsymbol{H}^{*}$ ) can increase to around 9 .

\section{SCATTERING BY MULTIPLE PLASMONIC CYLINDERS IN ARRAYS}

First in this section, we characterize the scattering by a single array of finite numbers of nanocylinders.

The nanocylinders considered are of the same composition each as given in Fig. 5(b). The other physical parameters such as the angle of incidence and amplitude of the incident wave are also the same as before. In Figs. 6(a)-6(c), total magnetic field intensity distributions of multiple cylinders arranged in an array with the number of cylinders varying from $2-4$ to 6 are depicted, respectively. The distance between two adjacent cylinders is $60 \mathrm{~nm}$. From these figures, one can see that the backscattering is strong due to the single array of plasmonic nanocylinders, but the peak values of the magnetic field intensity decrease as compared to that shown earlier in Fig. 5(b). This is attributed to the mutual coupling effects among the multiple plasmonic nanoparticles and the resonance spreading of the conserved energy. From these figures, it is also clearly seen that the resonances occur at the two ends of the single array regardless of the number of nanocylinder array elements. This is why we did not extend the characterization to a periodic array with a larger number of elements, when a normal incidence of a plane wave is considered.

In Fig. 7, the field intensity distribution is depicted for a double array of cylinders. The four nanocylinders are located at the corner points of square $(x= \pm 30 \mathrm{~nm}, y= \pm 30 \mathrm{~nm})$ in Fig. 7(a).

In Figs. 7(b) and 7(c), we extend the double array periodically, where the distance between two adjacent cylinders is a constant of $60 \mathrm{~nm}$ and the two single arrays are separated by a distance of $60 \mathrm{~nm}$. It is seen that compared to the results shown in Fig. 6, the backscattering of the near-zone magnetic field intensity is slightly stronger in Fig. 7, which is mainly due to the coupling effects of the nanocylinder arrays.

When the distance between two arrays increases, the coupling between the two arrays will become weaker so that the scattered fields due to each of the arrays will be localized around the nanocylinder arrays in their own near-field regions. This observation is shown in Fig. 8.

In the calculations, the two arrays shown in Figs. 8(a)-8(c) are (a) $140 \mathrm{~nm}$, (b) $200 \mathrm{~nm}$, and (c) $300 \mathrm{~nm}$, respectively, apart from each other. When the two single arrays are closer, the mutual coupling between them is strong so that the second array is also excited at resonance in the middle as depicted in Fig. 8(a). When the distance between the two arrays is appropriately controlled, say, $200 \mathrm{~nm}$ as shown in Fig. 8(b), the first single array of nanocylinders is excited at its resonance modes while the second single array is not. In this case, the mutual coupling between the two single arrays leads the field distribution along the whole single array to be significantly enhanced. When the distance between the two arrays is far apart, the mutual coupling is very weak, so each of the single array is considered as a localized array. As shown in Fig. 6(c) where the distance
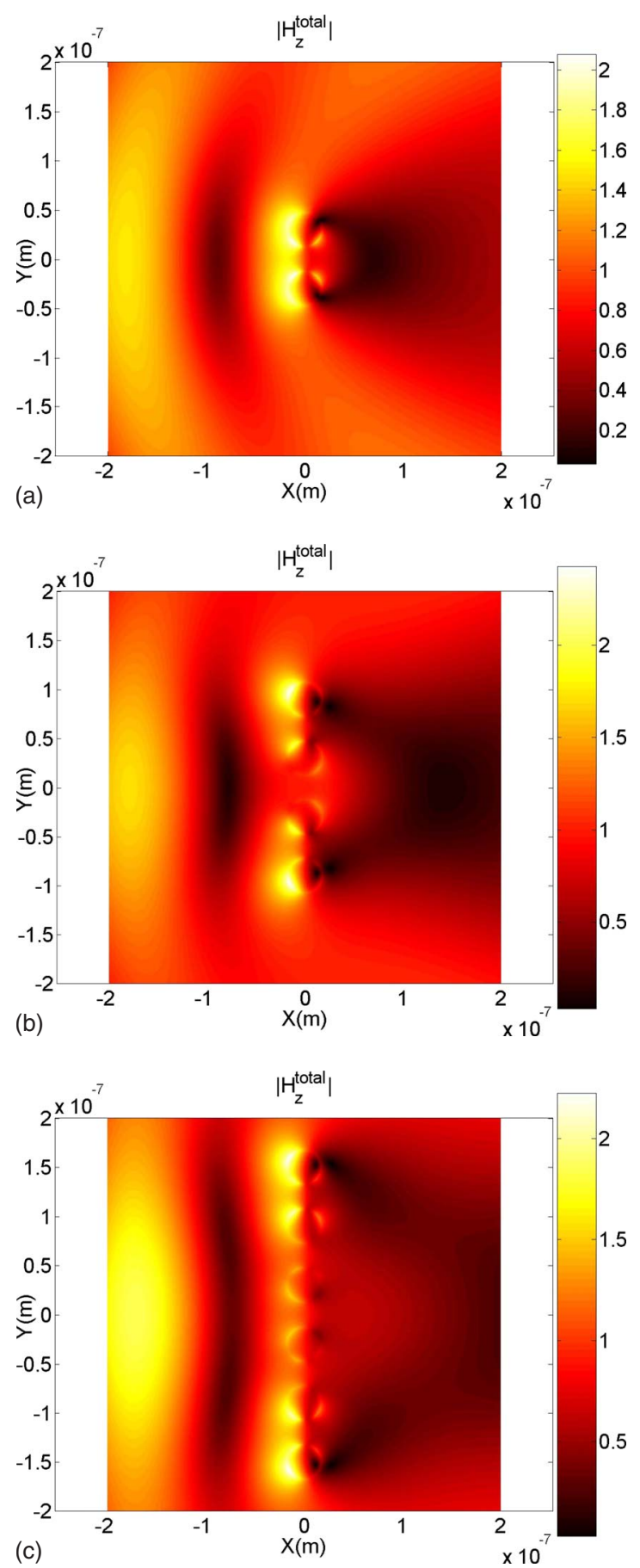

FIG. 6. (Color online) Scattering by a single array with different numbers of identical plasmonic resonant cylinders. The spacing between two adjacent cylinders is $60 \mathrm{~nm}$ and radii of the cylinders are $17.5 \mathrm{~nm}$.

between the two arrays is $300 \mathrm{~nm}$, the single array effects discussed earlier in Fig. 6 are observed. In this case, the resonances occur at the two ends of the first single array while the second array is not excited at its resonance.

\section{SCATTERING BY MULTIPLE PLASMONIC CYLINDERS WITH DIFFERENT DISTRIBUTIONS}

From the figures shown in SecIV, we find that the backscattering of an array of nanocylinders is strong but the peak resonance level of the total magnetic field is not as high as that of a single nanoparticle shown in Fig. 5(b). So in this 

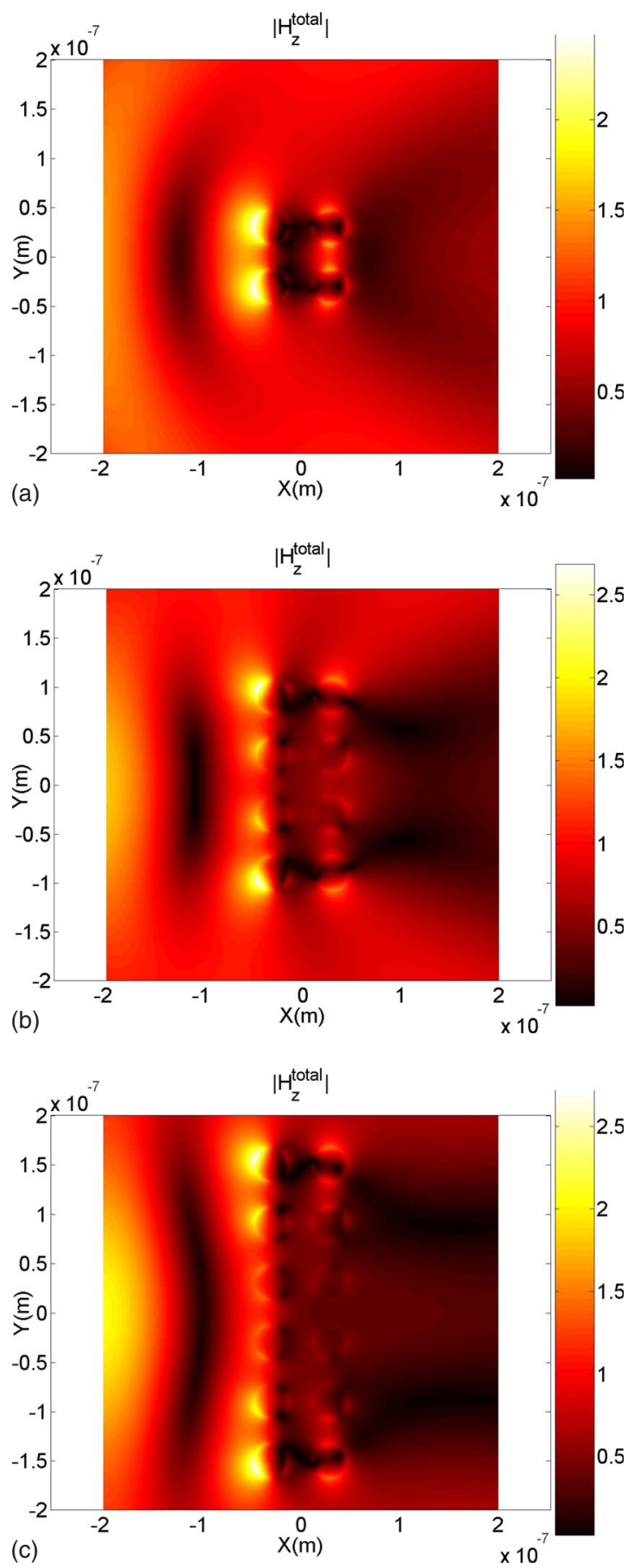

FIG. 7. (Color online) Scattering by a double array with different numbers of identical resonant plasmonic cylinders. The spacing between two adjacent cylinders and the distance between two single arrays are both $60 \mathrm{~nm}$.

section we will propose some structures consisting of multiple plasmonic nanocylinders which can enhance the backscattered field intensity values.

Plotted in Fig. 9 is the magnetic field intensity distribution of TE plane wave scattered by multiple cylinders. A system of three plasmonic cylinders depicted in Fig. 9(a) is considered first and its elements are located at $(20,0 \mathrm{~nm})$, $(85,55 \mathrm{~nm})$, and $(85,-55 \mathrm{~nm})$, respectively. The system can be considered as a single nanocylinder backed by a single array of two nanocylinders. It can be observed that the peak value of the total field is over 4. Apparently, the backscatter-
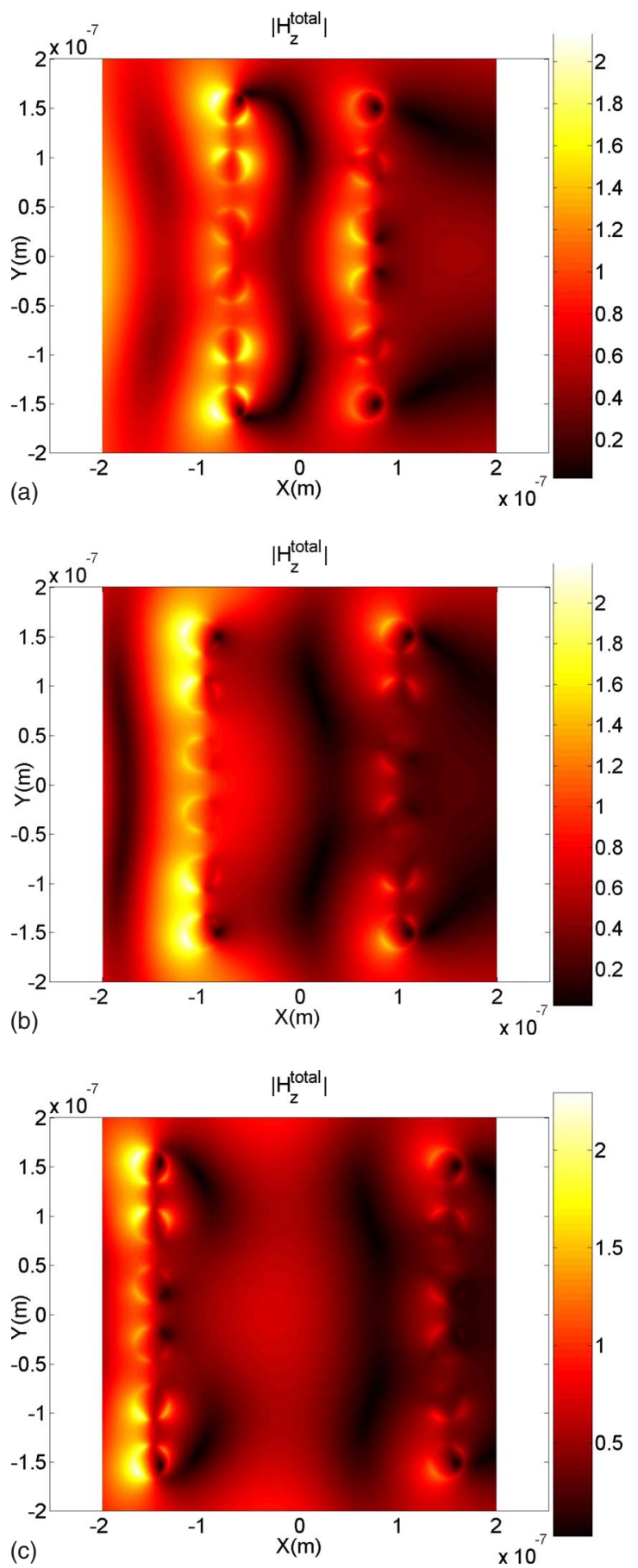

FIG. 8. (Color online) Scattering by two arrays of identical plasmonic resonant cylinders. The spacing between two adjacent cylinders is $60 \mathrm{~nm}$, but the distance between two single arrays varies from (a) $140 \mathrm{~nm}$ to (b) $200 \mathrm{~nm}$, to (c) $300 \mathrm{~nm}$.

ing is enhanced significantly, as compared to the result for a single nanocylinder shown in Fig. 5(b). This is because of the coupling effects due to the other two cylinders behind it. In Fig. 9(b), the number of the backed array elements or nanocylinders increases to four to make a total number of five nanocylinders. It is seen that the peak value of the field intensity of the single nanocylinder backed by a single array of four nanocylinders can be enhanced up to about 4.3. To see the effects of the array shapes, the field intensity of a triangle configuration composed of six nanocylinders is de- 

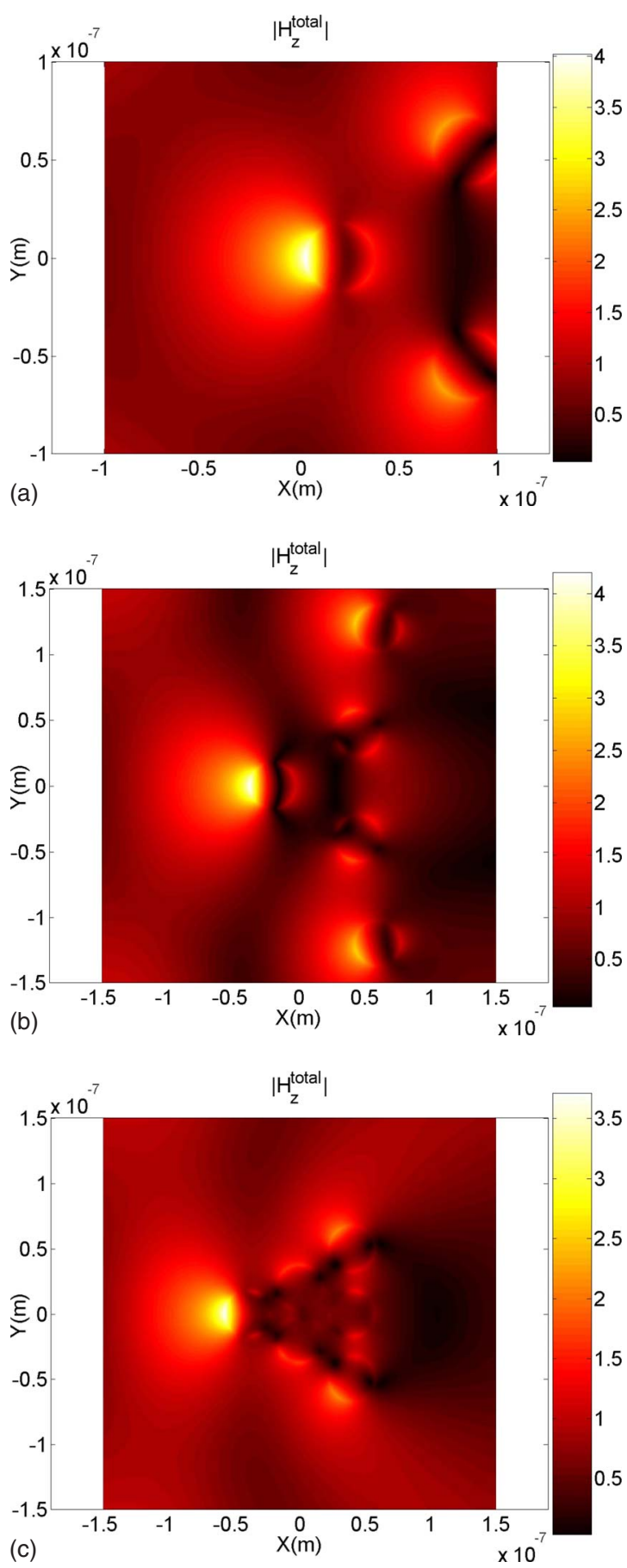

FIG. 9. (Color online) Scattering by multiple plasmonic nanocylinders. The near-field magnetic intensity distribution is shown. Significant enhancement in backscattering can be observed.

picted in Fig. 9(c). The total field intensity is still stronger than that in Fig. 6(b), but it is weaker than those shown in Figs. 9(a) and 9(b) because the adjacent cylinders are too close to each other so the mutual coupling is too strong.

\section{CONCLUSIONS}

Scattering coefficients and optical properties of a single nanocylinder array and a double nanocylinder array are theoretically formulated and numerically calculated; and the near-field magnetic field intensity distributions for various multiple nanocylinder systems are discussed in detail. Spe- cifically, plasmonic nanocylinders with dissipation are considered and significant enhancement in backscattering is observed from the theoretically and numerically obtained data. From various case studies, some physical insight is obtained and also design principles are demonstrated. The results obtained here to enhance the scattered energy in near-field region are important for the optical sensors and imaging, and quite essential for their other potential attractive applications in nanoscience and nanotechnology.

\section{ACKNOWLEDGMENTS}

The authors are grateful for the support in part by U.S. Air Force Projects (Grant Nos. AOARD-064031 and AOARD-074024) and a Research Grant of Academic Research Funds by the National University of Singapore. The support to L.-W.Li in terms of an Invited Visiting Professorship by Swiss Federal Institute of Technology, Lausanne, Switzerland is also much appreciated.

${ }^{1}$ D. Felbacq, G. Tayeb, and D. Maystre, J. Opt. Soc. Am. A 11, 2526 (1994).

${ }^{2}$ J. Yang, L. W. Li, and C. H. Liang, IEEE Antennas Wireless Propag. Lett. 2, 18 (2003).

${ }^{3}$ J. Yang, L. W. Li, K. Yasumoto, and C. H. Liang, IEEE Trans. Geosci. Remote Sens. 43, 280 (2005).

${ }^{4} \mathrm{C}$. W. Chew, Waves and Fields in Inhomogeneous Media (Van Nostrand Reinhold, New York, 1990).

${ }^{5}$ K. Yasumoto and K. Yoshitomi, IEEE Trans. Antennas Propag. 47, 1050 (1999).

${ }^{6}$ Y. Li, C. Wang, N. Zhang, C. Y. Wang, and Q. Xing, Appl. Opt. 45, 8462 (2006).

'B. S. Luk'yanchuk and V. Ternovsky, Phys. Rev. B 73, 235432 (2006).

${ }^{8}$ Z. L. Wang, Y. Q. Jin, and H. Ogura, Phys. Rev. B 71, 155415 (2005).

${ }^{9}$ Z. Chen, X. Li, A. Taflove, and V. Backman, Appl. Opt. 45, 633 (2006).

${ }^{10}$ R. Atkinson, W. R. Hendren, G. A. Wurtz, W. Dickson, A. V. Zayats, P. Evans, and R. J. Pollard, Phys. Rev. B 73, 235402 (2006).

${ }^{11}$ G. Gumbs and G. R. Aizin, Phys. Rev. B 65, 195407 (2002).

${ }^{12}$ A. Chen, S. J. Chua, X. H. Zhang, and J. R. Dong, Appl. Phys. Lett. 90, 011113 (2007).

${ }^{13}$ U. Schroter and A. Dereux, Phys. Rev. B 64, 125420 (2001).

${ }^{14}$ E. Devaux, A. Dereux, E. Bourillot, J. Weeber, Y. Lacroute, J. Goudonnet, and C. Girard, Phys. Rev. B 62, 10504 (2000).

${ }^{15}$ J. M. Steele, C. E. Moran, A. Lee, C. M. Aguirre, and N. J. Halas, Phys. Rev. B 68, 205103 (2003).

${ }^{16}$ T. Ochiai and J. Sánchez-Dehesa, Phys. Rev. B 65, 245111 (2002).

${ }^{17}$ A. L. Pokrovsky and A. L. Efros, Phys. Rev. B 65, 045110 (2002).

${ }^{18}$ G. Ding, C. T. Chan, Z. Q. Zhang, and P. Sheng, Phys. Rev. B 71, 205302 (2005).

${ }^{19}$ J. Song, R. P. Zaccaria, M. B. Yu, and X. W. Sun, Opt. Express 14, 8812 (2006).

${ }^{20}$ M. Laroche, S. Albaladejo, R. Gómez-Medina, and J. J. Sáenz, Phys. Rev. B 74, 245422 (2006).

${ }^{21}$ E. Centeno and D. Felbacq, J. Opt. Soc. Am. B 23, 2257 (2006).

${ }^{22}$ F. I. Baida, A. Belkhir, and D. V. Labeke, Phys. Rev. B 74, 205419 (2006).

${ }^{23}$ D. Dai, Y. Shi, and S. He, Appl. Opt. 45, 4941 (2006).

${ }^{24}$ E. Popov, M. Nevière, S. Enoch, and R. Reinisch, Phys. Rev. B 62, 16100 (2000).

${ }^{25}$ M. I. Haftel, C. Schlockermann, and G. Blumberg, Appl. Phys. Lett. 88, 193104 (2006).

${ }^{26}$ R. Gómez-Medina, M. Laroche, and J. J. Sáenz, Opt. Express 14, 3730 (2006).

${ }^{27}$ H. S. Chu, W. B. Ewe, E. P. Li, and R. Vahldieck, Opt. Express 15, 4216 (2007).

${ }^{28}$ L.-S. Chen, C.-H. Kuo, and Z. Ye, Phys. Rev. E 69, 066612 (2004).

${ }^{29}$ Z. Zhang, J. Du, X. Guo, X. Luo, and C. lei Du, J. Appl. Phys. 102, 074301 (2007).

${ }^{30}$ S. Fan and J. D. Joannopoulos, Phys. Rev. B 65, 235112 (2002).

${ }^{31}$ E. Moreno, L. Martín-Moreno, and F. J. García-Vidal, J. Opt. A, Pure Appl. Opt. 8, S94 (2006). 
${ }^{32}$ G. Shvets and Y. A. Urzhumov, J. Opt. A, Pure Appl. Opt. 8, S122 (2006).

${ }^{33}$ B. C. Gupta and Z. Ye, J. Appl. Phys. 94, 2173 (2003).

${ }^{34}$ B. C. Gupta and Z. Ye, Phys. Rev. B 67, 153109 (2003).

${ }^{35}$ C. Kuo and Z. Ye, Phys. Rev. E 70, 026608 (2004).

${ }^{36}$ C. Sohl, C. Larsson, M. Gustafsson, and G. Kristensson, J. Appl. Phys. 103, 054906 (2008).

${ }^{37}$ T. Li, S.-M. Wang, H. Liu, J.-Q. Li, F.-M. Wang, and S.-N. Zhu, J. Appl. Phys. 103, 023104 (2008).

${ }^{38}$ A. A. Zharov and N. A. Zharova, J. Appl. Phys. 103, 013109 (2008).

${ }^{39}$ J. Li, M.-H. Lu, T. Fan, X.-K. Liu, L. Feng, Y.-F. Tang, and Y.-F. Chen, J. Appl. Phys. 102, 073538 (2007).

${ }^{40} \mathrm{H}$. Li, Y. Zhang, L. Zhang, L. He, H. Li, and H. Chen, J. Appl. Phys. 102, 033711 (2007).

${ }^{41}$ B.-I. Wu, J. Lu, J. A. Kong, and M. Chen, J. Appl. Phys. 102, 114907
(2007).

${ }^{42}$ A. Baev, E. P. Furlani, M. Samoc, and P. N. Prasad, J. Appl. Phys. 102, 043101 (2007).

${ }^{43}$ G. X. Li, H. L. Tam, F. Y. Wang, and K. W. Cheah, J. Appl. Phys. 102, 116101 (2007).

${ }^{44}$ C. Kuo and Z. Ye, Phys. Rev. E 70, 056608 (2004).

${ }^{45}$ C. W. Qiu, H. Y. Yao, L. W. Li, S. Zouhdi, and T. S. Yeo, Phys. Rev. B 75, 245214 (2007)

${ }^{46}$ C. W. Qiu, H. Y. Yao, L. W. Li, S. Zouhdi, and T. S. Yeo, Phys. Rev. B 75, 155120 (2007).

${ }^{47}$ H. Y. She, L. W. Li, O. J. F. Martin, and J. R. Mosig, Opt. Express 16, 1007 (2008).

${ }^{48}$ H. Caglayan, I. Bulu, and E. Ozbay, J. Appl. Phys. 103, 053105 (2008). 